\section{FATORES DE RISCO CARDIOVASCULAR E PREVALÊNCIA DE SÍNDROME METABÓLICA EM IDOSOS}

\author{
Cardiovascular risk factors and prevalence of metabolic \\ syndrome in the elderly
}
Factores de riesgo cardiovascular y prevalencia del síndrome metabólico en mayores

\section{RESUMO}

Objetivo: Avaliar a prevalência da síndrome metabólica (SM) em idosos atendidos em uma Unidade Básica de Saúde (UBS) e a relação entre medidas antropométricas, hemodinâmicas e bioquímicas. Métodos: Estudo transversal com 94 idosos atendidos na UBS Cecília de Sousa Neri, em Picos-PI, no período de agosto a outubro de 2011. Foram avaliados peso, altura, circunferência da cintura (CC), pressão arterial sistólica (PAS) e diastólica (PAD); além disso, coletou-se informação sobre dados bioquímicos. Considerouse síndrome metabólica (SM) quando presentes três ou mais fatores de risco, segundo o National Cholesterol Education Program. Realizou-se o teste $t$ e de Mann-Whitney, para comparação de médias entre os sexos, correlação de Pearson entre variáveis antropométricas, hemodinâmicas e bioquímicas, e associação entre as variáveis pelo teste qui-quadrado, com nível de significância de 5\%. Resultados: Amostra com 68,1\% $(\mathrm{n}=64)$ de mulheres. A média de CC foi de 102,4 $\pm 11,4 \mathrm{~cm}$ (homens) e 96,2 $\pm 13,2 \mathrm{~cm}$ (mulheres), ( $\mathrm{p}=0,031)$. A SM foi diagnosticada em $38,64 \%(n=34)$ dos pesquisados, sendo de $28,40 \%(n=25)$ para as mulheres $(p=0,252)$. Houve correlação significativa entre peso e PAS $(r=0,273 ; p=0,0076)$, peso e PAD $(r=0,379 ; p=0,0002), C C$ e PAS $(r=0,248 ; p=0,0158), C C$ e PAD $(r=0,255 ; p=0,0129)$, IMC e PAS $(r=0,294 ; p=0,0041)$, IMC e PAD $(r=0,318 ; p=0,0018)$, peso e glicemia $(r=0,184$; $\mathrm{p}=0,0855)$, PAD e glicemia $(\mathrm{r}=0,219 ; \mathrm{p}=0,0403)$. Conclusão: Houve elevada prevalência de SM, além de relações positivas entre variáveis antropométricas, glicemia, triglicerídeos e pressão arterial, o que mostra a necessidade de monitoramento e controle desses fatores de risco cardiovascular.

Descritores: Pressão Arterial; Glicemia; Antropometria; Doença Crônica.

\section{ABSTRACT}

Objective: To evaluate the prevalence of metabolic syndrome (MS) in elderly patients seen at a Basic Health Unit (BHU) and the relationship between anthropometric, hemodynamic and biochemical measures. Methods: Cross-sectional study with 94 elderly seen at Cecilia de Sousa Neri UBS, in Picos, Piaui, in the period from August to October 2011. Weight, height, waist circumference (WC), and systolic (SBP) and diastolic (DBP) blood pressure were evaluated, and biochemical data was collected. The metabolic syndrome was characterised by the presence of three or more risk factors, in accordance with the National Cholesterol Education Program. The study applied t-test and Mann-Whitney test for comparison of means between the sexes, Pearson's correlation between the anthropometric, hemodynamic and biochemical variables, and associations between variables using chi-square test, with 5\% significance level. Results: Of the sample, $68.1 \%(n=64)$ were women. Mean WC was $102.4 \pm 11.4 \mathrm{~cm}$ (males) and $96.2 \pm 13.2 \mathrm{~cm}$ (females), $(p=0.031) . M S$ was diagnosed in $38.64 \%(n=34)$ of the participants, being $28.40 \%(n=25)$ for women $(p=0.252)$. There was a significant correlation between weight and $S B P(r=0.273 ; p=0.0076)$, weight and $D B P(r=0.379 ; p=0.0002), W C$ and $\operatorname{SBP}(r=0.248 ; p=0.0158), W C$ and $D B P(r=0.255$; $p=0.0129)$, BMI and SBP $(r=0.294 ; p=0.0041), B M I$ and DBP $(r=0.318 ; p=0.0018)$, weight and blood glucose $(r=0.184 ; p=0.0855)$ and DBP and blood glucose $(r=0.219 ; p=0.0403)$.
Artigo Original
1) Universidade Federal do Piauí - UFPI Teresina (PI) - Brasil
Recebido em: 21/01/2014 Revisado em: 03/08/2014 Aceito em: 19/10/2014 
Conclusion: There was high prevalence of $M S$, as well as positive relationships between the anthropometric variables, blood glucose, triglycerides and arterial blood pressure, showing the need for monitoring and control of these cardiovascular risk factors.

Descriptors: Blood Pressure; Blood Glucose; Anthropometry; Chronic Disease.

\section{RESUMEN}

Objetivo: Evaluar la prevalencia del síndrome metabólico (SM) en mayores asistidos en una Unidad Básica de Salud (UBS) y la relación entre medidas antropométricas, hemodinámicas y bioquímicas. Métodos: Estudio transversal con 94 mayores asistidos en la UBS Cecília de Sousa Neri, en Picos-PI, entre agosto y octubre de 2011. Fueron evaluados el peso, la altura, la circunferencia de la cintura (CC), la presión arterial sistólica (PAS) y diastólica (PAD); además, se recogió informaciones de los datos bioquímicos. Se consideró el síndrome metabólico (SM) cuando se identificaba tres o más factores de riesgo según el National Cholesterol Education Program. Se realizó la prueba $t$ y de Mann-Whitney para comparación de medias entre los sexos, correlación de Pearson entre las variables antropométricas, hemodinámicas y bioquímicas y asociación de las variables a través de la prueba del Chi-cuadrado con nivel de significancia del 5\%. Resultados: Muestra con el 68,1\% (n=64) de mujeres. La media de CC fue de 102,4 $\pm 11,4 \mathrm{~cm}$ (hombres) y 96,2 $\pm 13,2 \mathrm{~cm}$ (mujeres), ( $p=0,031)$. El SM fue diagnosticado en el 38,64\% ( $n=34)$ de los investigados con un $28,40 \%(n=25)$ en las mujeres $(p=0,252)$. Hubo correlación significativa entre el peso y la PAS ( $r=0,273$; $p=0,0076)$, el peso y la PAD ( $r=0,379$; $p=0,0002)$, la CC y la PAS ( $r=0,248 ; p=0,0158)$, la CC y la PAD $(r=0,255 ; p=0,0129)$, el IMC y la PAS ( $r=0,294 ; p=0,0041)$, el IMC y la PAD $(r=0,318 ; p=0,0018)$, el peso y la glucosa $(r=0,184$; $p=0,0855)$, la PAD y la glucosa $(r=0,219 ; p=0,0403)$. Conclusión: Hubo elevada prevalencia del SM, además de relaciones positivas entre las variables antropométricas, glucemia, triglicéridos y presión arterial lo que sugiere la necesidad del control de esos factores de riesgo cardiovascular.

Descriptores: Presión arterial; Glucemia; Antropometría; Enfermedad Crónica.

\section{INTRODUÇÃO}

O aumento da população de idosos no Brasil nos últimos anos é decorrente do processo de envelhecimento populacional, que ocorre de forma progressiva e inevitável com o avanço da idade, independentemente do estilo de vida, dos fatores ambientais ou das doenças. Esse processo acarreta alterações morfofisiológicas, propiciando maior incidência de processos patológicos ${ }^{(1)}$.

De acordo com as Nações Unidas, em 2011, a população idosa correspondia a $11 \%$ da população mundial, e em 2050 , corresponderá a $22 \%{ }^{(2)}$. Portanto, um contingente maior de pessoas está alcançando idades mais avançadas e vivendo mais anos, embora com maior incapacidade. Benefícios do sistema de infraestrutura, saneamento e habitação, bem como mudanças sociais na área de educação, percepção e comportamento, ligados à área de saúde e nutrição, têm sido relacionados ao aumento na longevidade da população mundial, com consequente aumento do número de idosos ${ }^{(3,4)}$.

Diante desse panorama mundial de rápido envelhecimento populacional e prolongamento da vida, os desafios estão estabelecidos. O principal deles é quanto à prevenção e promoção da saúde, além de medidas de tratamento quando uma parcela desse contingente de idosos apresentar algum tipo de doença e/ou incapacidade ${ }^{(3)}$.

Assim, o desafio não é prolongar a vida sem melhoria da qualidade de vida, e sim dispor de adequada assistência, prevenção e promoção da saúde não somente para as idades mais tenras, mas também na fase de envelhecimento, evitando mortes prematuras de idosos, haja vista que esse grupo de indivíduos apresenta maior vulnerabilidade à doença, sendo estes muitas vezes acarretados por doenças crônico-degenerativas, como a síndrome metabólica ${ }^{(1,3)}$.

A síndrome metabólica (SM) é um conjunto de fatores que, associados, aumentam o risco de problemas cardiovasculares. Esses fatores são: obesidade; aumento da circunferência abdominal; pressão arterial elevada; alterações de colesterol, triglicérides e glicemia. Ressaltando que, para fazer o diagnóstico, é preciso que o paciente tenha três dos cinco fatores de risco citados $^{(5)}$. Atualmente, essa síndrome é um dos maiores desafios para a saúde pública, por estar associada às doenças cardiovasculares, a principal causa de morte em todo o mundo ${ }^{(1)}$.

Os idosos são o grupo da população com maior prevalência de eventos cardiovasculares. Sendo assim, identificar a prevalência da síndrome metabólica entre eles é de grande importância para medidas de controle dos riscos. Além disso, existe um maior risco para déficits cognitivos entre os portadores dessa síndrome, em especial quando a glicemia alterada é um dos componentes ${ }^{(4,6)}$.

O presente estudo teve por objetivo avaliar a prevalência da síndrome metabólica em idosos atendidos em uma Unidade Básica de Saúde (UBS) e a relação entre medidas antropométricas, hemodinâmicas e bioquímicas.

\section{MÉTODOS}

Tratou-se de estudo do tipo quantitativo e transversal, realizado em idosos com idade superior a 60 anos atendidos em uma UBS, participantes do Programa Hiperdia, da Estratégia de Saúde da Família, desenvolvido no período de agosto a outubro de 2011. 
De acordo com a Secretaria Municipal de Saúde, essa UBS da cidade de Picos-PI possui 561 idosos cadastrados, sendo 310 do sexo feminino e 251, do sexo masculino. Porém a amostra final $(\mathrm{n}=94)$ desta pesquisa representou $16,8 \%$ do total, formada por conveniência, de acordo com a demanda da UBS no período estabelecido para coleta. Excluíram-se os idosos com presença de incapacidade física, hepatopatias, esteatose hepática e câncer.

Para avaliação do estado nutricional, foram coletadas medidas de peso, altura e circunferência da cintura. A aferição de peso e altura foi realizada por um avaliador treinado, seguindo a padronização ${ }^{(7)}$. Realizou-se uma medida de peso e três de altura, sendo considerada a média dos valores. Para a realização das medidas de circunferência da cintura, utilizou-se fita métrica inelástica e flexível de 150 $\mathrm{cm}$ de comprimento, com precisão de uma casa decimal, obedecendo à padronizaçãa ${ }^{(8,9)}$. A aferição aconteceu com o indivíduo em pé, em posição ereta, com abdômen relaxado, braços dispostos ao longo do corpo e pés juntos.

Verificou-se a pressão arterial (PA) em um único momento, em triplicata, no braço esquerdo, com esfignomanômetro Premium ${ }^{\circledR}$. Antes da medida, cada sujeito ficou sentado por 20 minutos, sendo a medida realizada nessa posição. Os valores médios que permitem classificar os indivíduos adultos acima de 18 anos foram de acordo com a VI Diretrizes Brasileiras de Hipertensão Arterial $(2010)^{(10)}$.

A partir dos valores de peso e altura, calculou-se o Índice de Massa Corporal (IMC), de acordo com a seguinte fórmula: peso/altura ${ }^{2}\left(\mathrm{~kg} / \mathrm{m}^{2}\right)$, sendo o estado nutricional classificado segundo os critérios para idosos: $<22 \mathrm{Kg} / \mathrm{m}^{2}$; eutrofia, valores entre 22 e $<27$; e sobrepeso, IMC de 27 $\mathrm{Kg} / \mathrm{m}^{2(11)}$.

Os parâmetros bioquímicos (glicemia, triglicerídeos) foram coletados utilizando-se de dados secundários, com resultado de exames realizados em período de até 3 meses anteriores à pesquisa. Esses dados foram anotados em formulário específico elaborado pelos pesquisadores. Ressalta-se que os exames foram realizados em laboratórios clínicos da cidade de Picos-PI, por meio de testes colorimétricos enzimáticos, utilizando kits adquiridos da Labtest (Belo Horizonte-MG), em equipamento modular da empresa Roche Diagnóstica. Os valores de referência para triglicerídeos foram definidos segundo a V Diretrizes Brasileiras de Dislipidemia e Prevenção da Aterosclerose ${ }^{(12)}$ e para glicemia de jejum, segundo o National Cholesterol Education Program $(2001)^{(5)}$.

Considerou-se síndrome metabólica quando presentes três ou mais das seguintes alterações nos idosos: obesidade abdominal (valores de circunferência de cintura superiores a $102 \mathrm{~cm}$ e $88 \mathrm{~cm}$, em homens e mulheres, respectivamente), hipertensão arterial sistêmica (níveis pressóricos $\geq$
130/85 mmHg), tolerância à glicose prejudicada (glicemia de jejum $\geq 110 \mathrm{mg} / \mathrm{dl}$ ) e hipertrigliceridemia (valores $\geq 150 \mathrm{mg} / \mathrm{dl})^{(5)}$.

Os dados foram apresentados como médias e desviospadrão. Foram realizados o teste $t \mathrm{e} o$ de Mann-Whitney para comparação de médias entre os sexos; correlação de Pearson entre variáveis antropométricas, hemodinâmicas e bioquímicas; e associação entre as variáveis pelo teste quiquadrado. Para tais análises, utilizou-se o software STATA 9.0, adotando-se o nível de significância $\mathrm{p}<0,05$.

O presente estudo foi submetido e aprovado pelo Comitê de Ética em Pesquisa da Universidade Federal do Piauí, em conformidade com a Resolução 466/12 do Conselho Nacional de Saúde (N. ${ }^{\circ}$ CAAE 0237.0.045.00011). Todos os que concordaram em participar assinaram o TCLE e foram observados todos os princípios éticos que regem a pesquisa científica envolvendo seres humanos.

\section{RESULTADOS}

No presente estudo, foram avaliados 94 idosos, sendo $68,1 \%(\mathrm{n}=64)$ do sexo feminino. A idade variou de 60 a 89 anos, com média de 71,7 $\pm 6,8$ anos e 70,5 $\pm 6,8$ anos para o sexo masculino e feminino, respectivamente (Tabela I).

Realizou-se análise de comparação de médias para idade, variáveis antropométricas e bioquímicas, segundo o sexo (Tabela I). O peso corporal, a altura e a circunferência da cintura foram estatisticamente diferentes entre os sexos $(\mathrm{p}<0,05)$. A média da circunferência da cintura (CC) foi estatisticamente maior para os homens $(p=0,031)$, se comparada à das mulheres $(96,2 \pm 13,2 \mathrm{~cm})(\mathrm{p}=0,031)$. Esses dados mostram que a população do estudo apresenta valores elevados de obesidade central. Observou-se, ainda, que a média do IMC da amostra foi de $26,8 \pm 3,6 \mathrm{~kg} / \mathrm{m}^{2}$ (homens) e $26,3 \pm 5,2 \mathrm{~kg} / \mathrm{m}^{2}$ (mulheres), sem diferença significativa entre os sexos, e classificados como eutróficos (Tabela I).

As médias dos valores pressóricos da pressão arterial sistólica (PAS) apresentaram valores maiores que os indicados na referência para SM, e as médias da pressão arterial diastólica (PAD) estavam com valores limítrofes referentes a essa recomendação. Com relação aos triglicerídeos do grupo, observou-se uma média dos valores elevada para ambos os sexos, com valor desejável inferior a $150 \mathrm{mg} / \mathrm{dl}$. Quanto à glicemia, pode-se destacar que o grupo pesquisado apresenta valores médios limítrofes em comparação ao recomendado, conforme descrição de valores na Tabela I.

A prevalência de síndrome metabólica foi diagnosticada em $38,64 \%(n=34)$ dos idosos pesquisados, com maior proporção para o sexo feminino $(28,40 \% ; n=25)$, mas sem diferença entre os sexos (Tabela II). 
Tabela I - Diferença entre as médias de idade, variáveis antropométricas e bioquímicas dos idosos segundo o sexo. TeresinaPI, 2011.

\begin{tabular}{lccc}
\hline Variáveis & Masculino (n=29) & Feminino (n=65) & $\boldsymbol{p}$ \\
\hline Idade (anos) & $71,7(6,8)$ & $70,5(6,9)$ & 0,446 \\
Peso (Kg) & $71,9(12,7)$ & $62,7(13,0)$ & $0,002^{\#}$ \\
Altura (cm) & $163,6(8,1)$ & $154,3(5,6)$ & $0,000^{\#}$ \\
Circunferência da cintura (cm) & $102,4(11,4)$ & $96,2(13,2)$ & $0,031^{\#}$ \\
Índice de Massa Corpórea (Kg/m²) & $26,8(3,6)$ & $26,3(5,2)$ & 0,649 \\
PAS (mmHg)* & $136,9(14,2)$ & $135,2(16,5)$ & 0,457 \\
PAD (mmHg)* & $83,4(10,8)$ & $84,1(10,4)$ & 0,621 \\
Triglicerídeos (mg/dL)* & $181,5(128,4)$ & $173,6(79,9)$ & 0,691 \\
Glicemia (mg/dL)* & $109,2(42,9)$ & $104,8(45,8)$ & 0,794 \\
\hline
\end{tabular}

Pressão arterial diastólica (PAD); Pressão arterial sistólica (PAS); Dados apresentados como média (desvio-padrão). (*) Teste de MannWhitney; demais variáveis, teste $t$ de Student. $\left(^{(}\right) \mathrm{p}<0,05$.

Tabela II - Prevalência de síndrome metabólica dos idosos atendidos em Unidade de Saúde, por sexo. Teresina-PI, 2011.

\begin{tabular}{|c|c|c|c|c|c|c|c|}
\hline \multirow{2}{*}{ Classificação } & \multicolumn{2}{|c|}{ Masculino } & \multicolumn{2}{|c|}{ Feminino } & \multicolumn{2}{|c|}{ Total } & \multirow{2}{*}{$p^{*}$} \\
\hline & n & $\%$ & n & $\%$ & n & $\%$ & \\
\hline Não possui SM & 17 & 19,32 & 37 & 42,05 & 54 & 61,36 & 0,252 \\
\hline Possui SM & 9 & 10,23 & 25 & 28,40 & 34 & 38,64 & \\
\hline Total & 26 & 29,55 & 62 & 70,45 & 88 & 100 & \\
\hline
\end{tabular}

Síndrome Metabólica (SM); $\left(^{*}\right)$ Teste qui-quadrado.

Tabela III - Classificação do risco de complicações metabólicas avaliados pela circunferência da cintura, de acordo com sexo e faixa etária. Teresina-PI, 2011.

\begin{tabular}{|c|c|c|c|c|c|c|c|c|}
\hline \multirow[t]{3}{*}{ Variáveis } & \multicolumn{8}{|c|}{ Risco de complicações metabólicas associadas à obesidade } \\
\hline & \multicolumn{2}{|c|}{ Normal } & \multicolumn{2}{|c|}{ Elevado } & \multicolumn{2}{|c|}{ Muito elevado } & \multicolumn{2}{|c|}{ Total } \\
\hline & $\mathbf{n}$ & $\%$ & $\mathbf{n}$ & $\%$ & $\mathbf{n}$ & $\%$ & n & $\%$ \\
\hline \multicolumn{9}{|l|}{ Sexo } \\
\hline Masculino & 8 & 27,59 & 5 & 17,24 & 16 & 55,17 & 30 & 100 \\
\hline Feminino & 7 & 9,38 & 9 & 14,06 & 49 & 76,56 & 64 & 100 \\
\hline \multicolumn{9}{|l|}{ Faixa etária } \\
\hline $60-69$ anos & 8 & 18,18 & 9 & 20,45 & 27 & 61,36 & 45 & 100 \\
\hline $70-79$ anos & 4 & 11,11 & 5 & 13,89 & 27 & 75,00 & 36 & 100 \\
\hline$>80$ anos & 3 & 15,38 & 0 & 0,00 & 11 & 84,62 & 13 & 100 \\
\hline
\end{tabular}

Teste Exato de Fisher, $\mathrm{p}=0,04$ para sexo e circunferência da cintura; e $\mathrm{p}=0,34$ para faixa etária e circunferência da cintura. 
Na Tabela III, observou-se um risco de complicações metabólicas associadas à obesidade central, segundo sexo e faixa etária, avaliado por intermédio da circunferência da cintura. Do total de idosos do estudo, 69,15\% $(n=65)$ apresentaram circunferência da cintura muito elevada, indicando elevada prevalência de risco na população, não havendo diferença estatisticamente significativa entre as faixas etárias $(p=0,34)$. O sexo feminino apresentou uma maior prevalência de "risco muito elevado" de complicações metabólicas $(76,56 \%, n=49)$, comparado ao sexo masculino $(55,17 \% ; n=16)(p=0,04)$.
Foi realizada uma análise de correlação entre as variáveis antropométricas, hemodinâmicas e os parâmetros bioquímicos demonstrados na Tabela IV. Verificou-se que peso e pressão arterial (peso x PAS: $\mathrm{r}=0,27 ; \mathrm{p}=0,007 \mathrm{e}$ peso x PAD: $r=0,38 ; p=0,000)$, circunferência da cintura e pressão arterial (CC x PAS: $r=0,25 ; \mathrm{p}=0,016$ e CC x PAD: $\mathrm{r}=0,25 ; \mathrm{p}=0,013$ ), assim como IMC e pressão arterial (IMC x PAS: $r=0,29 ; p=0,004$ e IMC x PAD: $r=0,32 ; p=0,002)$ apresentaram correlações positivas e significativas. Não houve correlação entre as variáveis antropométricas e bioquímicas $(\mathrm{p}>0,05)$.

Tabela IV - Correlação entre variáveis antropométricas, hemodinâmicas e parâmetros bioquímicos. Teresina-PI, 2011.

\begin{tabular}{lcc}
\hline Variáveis & Correlação $(\mathbf{r})$ & $\boldsymbol{p}$ \\
\hline CC x Peso & 0,88 & $0,000^{*}$ \\
CC x IMC & 0,85 & $0,000^{*}$ \\
Peso x PAS & 0,27 & $0,007^{*}$ \\
Peso x PAD & 0,38 & $0,000^{*}$ \\
CC x PAS & 0,25 & $0,016^{*}$ \\
CC x PAD & 0,25 & $0,013^{*}$ \\
IMC x PAS & 0,29 & $0,004^{*}$ \\
IMC x PAD & 0,32 & $0,002^{*}$ \\
Peso x Glicose & 0,18 & 0,085 \\
PAD x Glicose & 0,22 & $0,040^{*}$ \\
\hline
\end{tabular}

Correlação de Pearson, $\mathrm{p}<0,05^{*}$. Circunferência da cintura (CC); Índice de Massa Corporal (IMC); Pressão arterial sistólica (PAS); Pressão arterial diastólica (PAD).

\section{DISCUSSÃO}

O envelhecimento da população, fenômeno globalmente observado, tem refletido no crescimento do impacto sobre a saúde, provocado pelas doenças crônicas e não transmissíveis. Dentre essas doenças, destaca-se a SM, que vem despertando, nas últimas décadas, o interesse da comunidade científica ${ }^{(12-14)}$.

Os achados mostram idosos com média de idade de aproximadamente 71 anos, IMC e CC elevadas para ambos os sexos, além de prevalência elevada de síndrome metabólica.

Observou-se, na literatura, uma ampla variação da prevalência de SM nas diferentes populações, de acordo com sexo, idade e critérios de classificação utilizados (International Diabetes Federation ou National Cholesterol Education Program - Adult Treatment Panel III), apesar de não existirem dados precisos acerca da prevalência nacional. As prevalências de SM encontradas em nossa amostra, avaliadas pela NCEP ATPIII, são elevadas, com maior prevalência de síndrome metabólica em mulheres ${ }^{(14-16)}$, o que pode ser justificado por elas tenderem a apresentar maior quantidade de gordura corporal. Com o avançar da idade, ocorre a redução dos níveis de estrogênio, com isso, há uma tendência para o desenvolvimento de dislipidemias e outras alterações metabólicas ${ }^{(17-19)}$.

Verificou-se, na presente pesquisa, uma elevada prevalência de risco de complicações cardiovasculares e metabólicas associadas à obesidade abdominal. No entanto, ao analisar os resultados de circunferência da cintura associada à idade, não foram observados dados estatisticamente significativos, mostrando que o risco de complicações relacionado à obesidade central é independente da idade no presente estudo, embora pesquisas ambulatoriais e de base populacional demonstrem aumento

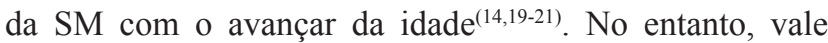
ressaltar que, na maioria desses estudos, a amostra incluiu indivíduos a partir de 18 anos, enquanto neste estudo, a partir de 60 anos.

Com relação às variáveis antropométricas e hemodinâmicas, percebeu-se que peso, CC e IMC se relacionaram positivamente com PAS e PAD, sendo possível sugerir que o excesso de peso com deposição central e generalizada de gordura são fatores predisponentes para a elevação da pressão arterial. Semelhante a esses achados, 
estudos demonstraram a relação entre aumento da massa corporal e elevação da pressão $\operatorname{arterial}^{(22-24)}$.

Os mecanismos pelos quais a obesidade se associa ao surgimento da hipertensão arterial inclui alteração metabólica como hiperinsulinemia e desequilibrio das adipocinas, sistema renina-angiotensina-aldosterona (SRAA) e sistema nervoso simpático ${ }^{(14,25,26)}$. A ativação do SRAA, na obesidade, aumenta a produção de angiotensina II, aldosterona, assim como outros metabólitos, potentes vasoconstrictores associados ao surgimento da hipertensão na obesidade. Além disso, a hiperinsulinemia na obesidade é um possível fator associado à hipertensão, pois a insulina induz a ativação do sistema nervoso central (SNC) com ação direta no rim por estimular a retenção de sódio $^{(26-28)}$. Porém, os dados dessa pesquisa mostram somente uma tendência de relação entre peso e alterações bioquímicas, como glicemia. Como a insulina não foi avaliada neste estudo e ela possui forte associação com os níveis de glicose, pode-se inferir que, com uma amostra maior, uma correlação positiva poderia ser observada entre a glicose de jejum e o peso corporal.

Apesar da limitada amostra do estudo, uma correlação positiva foi encontrada para a pressão arterial diastólica e glicose. Esses dados indicam a importância do controle de parâmetros bioquímicos e hemodinâmicos nessa população, pois existem evidências de que a elevação da glicose sanguínea está associada ao aumento do risco cardiovascular e à intolerância à glicose com o processo aterosclerótico $^{(25-28)}$.

O IMC dos idosos nesta pesquisa foi classificado segundo Lipstich ${ }^{(10)}$, cujo ponto de corte para sobrepeso/ obesidade é maior do que para a população adulta, usualmente utilizada pela OMS (1998), e mesmo assim, verificou-se um percentual elevado de sobrepeso no grupo investigado. Pesquisas demonstram que o ganho ponderal aumenta a ocorrência de fatores envolvidos na SM e implica em maior risco cardiovascular e metabólico ${ }^{(18,20,28)}$.

Desse modo, ressalta-se a relevância de avaliar a prevalência da SM e observar a relação dos diversos fatores de risco associados a eventos cardiovasculares, como alterações glicídicas, triglicerídeos elevados, excesso de peso, obesidade abdominal e aumento dos valores de pressão arterial sistêmica.

Os resultados identificados nesta pesquisa poderão subsidiar a formulação de programas de políticas e ações públicas eficazes para o controle dos fatores de risco associados à $\mathrm{SM}$, com incentivo à melhoria da qualidade de vida desses idosos.

Como limitação do estudo, deve ser ressaltado que o grupo pesquisado fazia parte do Programa Hiperdia, da Estratégia de Saúde da Família, portanto, obrigatoriamente formado por hipertensos e/ou diabéticos, os quais, consequentemente, são mais expostos e têm maior potencial de preencher os critérios diagnósticos da SM. Esse fato deve ser considerado como um viés de seleção nesta pesquisa e limitação do estudo.

\section{CONCLUSÃO}

Houve elevada prevalência de SM, sendo a maior entre as mulheres na amostra estudada, além de relações positivas entre variáveis antropométricas, bioquímicas e hemodinâmicas, o que mostra a necessidade de monitoramento e controle efetivo desses fatores de risco cardiovascular.

\section{AGRADECIMENTOS}

A Hildegardes Gomes de Medeiros Borges, da Secretaria Municipal de Saúde Picos.

\section{CONFLITO DE INTERESSE}

Sem conflito de interesse.

\section{REFERÊNCIAS}

1. Silva CG, Rodrigues JB, Medeiros Júnior JR, Coutinho NPS, Lopes MLH, Sardinha AHL. Perfil dos idosos com síndrome metabólica. Rev Pesq Saúde. 2012;13(2):1720.

2. United Nations, Department of Economic and Social Affairs. Population division: world population prospects: the 2012 revision. New York: United Nations; 2013.

3. Gragnolati $\mathrm{M}$, Jorgensen $\mathrm{OH}$, Rocha $\mathrm{R}$, Fruttero A. Growing old in an older Brazil: implications of population aging on growth, poverty, public finance and service delivery. Washington: The World Bank; 2011.

4. Camarano AA. Cuidados de longa duração para população idosa: um novo risco social a ser assumido? In: Hirata H., Guimarães NA, organizadores. Cuidado e cuidadoras: as várias faces do trabalho do care. $1^{\mathrm{a}} \mathrm{ed}$. São Paulo: Atlas, 2012. p. 148-65.

5. Expert Panel on Detection, Evaluation, and Treatment of High Blood Cholesterol in Adults. Executive summary of the third report of the National Cholesterol Education Program (NCEP) Expert Panel on Detection, Evaluation, and Treatment of High Blood Cholesterol in Adults (Adult Treatment Panel III). JAMA. 2001;285(19):2486-97. 
6. I-Te Lee, Yen-Feng Chiu, Chii-Min Hwu, Chih-Tsueng $\mathrm{He}$, Fu-Tien Chiang, Yu-Chun Lin et al. Central obesity is important but not essential component of the metabolic syndrome for predicting diabetes mellitus in a hypertensive family-based cohort. Results from the Stanford Asia-Pacific Program for Hypertension and Insulin Resistance (SAPPHIRe) Taiwan follow-up study. Cardiovasc Diabetol. 2012;11:43.

7. Eckel RH, Alberti KG, Grundy SM, Zimmet PZ. The metabolic syndrome. Lancet. 2010;375(9468):181-3.

8. Gordon CC, Chumlea WC, Roche AF. Stature recumbert, lenght, and weight. In: Lohman TG, Roche AF, Martorell R. Anthopometric standardization reference manual. Champaingn: Human Kinetics; 1988. p.3-8.

9. Callaway CW. Circumferences. In: Lohman TG, Roche AF, Martorell R, editors. Anthropometric standardization reference manual. Champaign: Human Kinetics Books, 1988. p. 39-54.

10. Sociedade Brasileira de Hipertensão. VI Diretrizes Brasileiras de Hipertensão. Arq Bras Cardiol. 2010;95(Supl 1):1-51

11. Lipschitz DA. Screening for nutritional status in the elderly. Primary Care.1994; 21(1):55-67.

12. Xavier HT, Izar MC, Faria Neto JR, Assad MH, Rocha VZ, Sposito AC, et al. V Diretriz Brasileira de Dislipidemias e Prevenção da Aterosclerose. Arq Bras Cardiol 2013; 101(4 Supl 1):1-22.

13. Cook NR, Paynter NP, Eaton CB, Manson JAE, Martin LW, Robinson JG, et al. Comparison of the Framingham and Reynolds Risk Scores for Global Cardiovascular Risk Prediction in the Multiethnic Women's Health Initiative. Circulation. 2012; 125(14):1748-56.

14. Moraes NS, Souza JAG, Miranda RD. Hipertensão arterial, diabetes mellitus e síndrome metabólica: do conceito à terapêutica. Rev Bras Hipertens. 2013;20(3):110-7.

15. Franco GPP, Scala LCN, Alves CJ, França GVA, Cassanelli Tjardim PCBV. Síndrome metabólica em hipertensos de Cuiabá-MT: prevalência e fatores associados. Arq Bras Cardiol. 2009;92(6):472-8.

16. Rigo JC, Vieira JL, Dalacorte RR, Reichert CL. Prevalência de síndrome metabólica em idosos de uma comunidade: comparação entre três métodos diagnósticos. Arq Bras Cardiol. 2009;93(2):85-91.

17. Felipe-de-melo ERT, Silva RCR, Assis AMO, Pinto EJ. Fatores associados à síndrome metabólica em trabalhadores administrativos de uma indústria de petróleo. Ciênc Saúde Coletiva. 2011;16(8):3443-52.

18. Kassim E, Pervanidou P, Kaltsas G, Chrousos G. Metabolic syndrome: definitions and controversies. BMC Med. 2011;9(1):1-13.

19. Lahsen R, Berry M. Surgical interventions to correct metabolic disorders. Br J Diabetes Vasc Dis. 2010;10(3):143-47.

20. Vlachopoulos C, Aznaouridis K, Stefanadis C. Prediction of cardiovascular events and all-cause mortality with arterial stiffness: a systematic review and meta-analysis. J Am Coll Cardiol. 2010; 55(13):131827.

21. Vanhoni LR, Xavier AJ, Piazza HE. Avaliação dos critérios de síndrome metabólica nos pacientes atendidos em ambulatório de ensino médico em Santa Catarina. Rev Bras Clin Med. 2012;10(2):100-5.

22. Simão $M$, Hayashida $M$, Santos $C B$, Cesarino EJ, Nogueira MS. Hipertensão arterial entre universitários da cidade de Lubango, Angola. Rev Latinoam Enferm. 2008;16(4):672-8.

23. Amira CO, Sokunbi DOB, Sokunbi A. The prevalence of obesity and its relationship with hypertension in an urban community: Data from world kidney day screening programme. Int $\mathrm{J}$ Med Biomed Res. 2012;1(2):104-10.

24. Martins MCC, Ricarte IF, Rocha CHL, Maia RB, Silva VB, Veras AB, et al. Pressão arterial, excesso de peso e nível de atividade física em estudantes de universidade pública. Arq Bras Cardiol. 2010;95(2):192-9.

25. Sarwar N, Gao P, Seshasai SR, Gobin R, Kaptoge $\mathrm{S}$, Di Angelantonio E, et al. Emerging risk factors collaboration. Diabetes mellitus, fasting blood glucose concentration, and risk of vascular disease: a collaborative meta-analyse of 102 prospective studies. Lancet 2010;375(9733):2215-22.

26. Kang YS. Obesity associated hypertension: new insights into mechanism. Electrolyte Blood Press 2013;11(2):46-52.

27. American Diabetes Association. Standards of medical care in Diabetes - 2013. Diabetes Care. 2013;36(Suppl 1):11-66.

28. Fox CS, Massaro JM, Hoffmann U, Pou KM, HorvatMaurovich P, Liu CY, et al. Abdominal visceral and subcutaneous adipose tissue compartments: association with metabolic risk factors in the Framingham Heart Study. Circulation, 2007;116(1):39-48. 


\section{Endereço do primeiro autor:}

José Filho da Silva

Departamento de Nutrição (UFPI)

Campus Universitário Ministro Petrônio Portella

Bairro: Ininga

CEP: 64049-550 - Teresina - PI - Brasil

E-mail:jfsaoju@hotmail.com

\section{Endereço para correspondência:}

Joilane Alves Pereira Freire

Departamento de Nutrição (UFPI)

Campus Universitário Ministro Petrônio Portella

Bairro: Ininga

CEP: 64049-550 - Teresina - PI - Brasil

E-mail: joilane@hotmail.com 